\title{
Detection of coherence-disrupting and coherence-conferring alterations in text
}

\author{
JAMSHED JAY BHARUCHA, KATHERINE L. OLNEY, and PAULA P. SCHNURR \\ Dartmouth College, Hanover, New Hampshire
}

\begin{abstract}
In a study phase, subjects were presented with sentence pairs which were either coherent or anomalous. In a subsequent recognition test, each sentence pair was presented either in its original form or with one word changed. The changed word either disrupted the coherence of a previously coherent sentence pair or conferred coherence on a previously anomalous sentence pair. Alterations were more accurately detected if they were coherence-disrupting than if they were coherence-conferring. In a coherence-conferring alteration, the altered sentence can be assimilated to one of the abstract cognitive units activated by the studied sentence pair, whereas in a coherence-disrupting alteration, the altered sentence cannot be so assimilated. Analogous asymmetries have been observed for musical sequences that are coherent or anomalous with respect to tonality and rhythm. These parallels suggest that principles of cognitive organization that are responsible for our intuitions of coherence cut across propositional and nonpropositional domains.
\end{abstract}

Text is one of many domains in which we have strong intuitions of coherence and anomaly. These intuitions are driven by internalized constraints on the ways in which events or states of affairs typically occur. A story's coherence typically hinges on the use of familiar settings, referents, causal relations, and motivations (Black, 1984). In a rather dissimilar domain, music, coherence typically hinges on the use of familiar organizations of tonal materials. Sentences in coherent text map onto unifying cognitive units which represent familiar plots (Lehnert, 1981), episodes (Rumelhart, 1977; Thorndyke, 1977), or sequences of episodes (Bower, Black, \& Turner, 1979; Schank \& Abelson, 1977). Elements in coherent music map onto unifying cognitive units which represent chord functions (Bharucha, 1984a), keys (Krumhansl \& Kessler, 1982), meters (Deutsch, 1983; Martin, 1972), rags (Bharucha, 1984b; Castellano, Bharucha, \& Krumhansl, 1984), among other structures. The intuition of coherence seems to stem from the organization of events under an abstract cognitive unit.

Although the criteria for coherence differ radically across domains, the discovery of associated memory phenomena that cut across domains would provide a robust basis for the convergence of these intuitions and would demonstrate a generality rather than a complete modularity of function. Similarities between memory for text and for music are particularly revealing because the representation of text is probably propositional, whereas the representation of music is probably not.

This research was supported in part by a Biomedical Research Support Grant from HHS (2-S07-RR07056-09). The authors thank Jack Baird, James Bartlett, Christa Bracci, Jay Dowling, Carol Fowler, Carol Krumhansl, Diana Raffman, Valerie Reyna, and George Wolford for helpful discussions. Requests for reprints should be sent to the first author, Department of Psychology, Dartmouth College, Hanover, NH 03755.
A familiar context provides a framework in which atypical (though not irrelevant) events are noticed and remembered, akin to the von Restorff effect (Bower et al., 1979). Unexpected events in an otherwise script-based story are easily detected (Bower et al., 1979), just as are "wrong" or sour notes in an otherwise tonal piece of music (Bharucha, 1984a). The relative salience of the unusual has been reported for other domains as well: faces (Cohen \& Carr, 1975; Light, Kayra-Stuart, \& Hollander, 1979), personality traits (Hastie \& Kumar, 1979), and roles and occupations (Woll \& Graesser, 1982). It should be noted, however, that the social and personality literature is equivocal on this point (see Berman, Read, \& Kenny, 1983; Johnson \& Judd, 1983; Woll \& Graesser, 1982).

Graesser, Gordon, and Sawyer (1979) found that, after subjects had read a script-based story, atypical foils were more easily detected than typical foils. Analogous effects have been observed for musical materials: An alteration of a tonal melody (one that conforms to the conventional constraints of tonal music) is more accurately detected if the tonal structure is violated than if it is preserved (Bharucha, 1984a; Cuddy, Cohen, \& Miller, 1979; Dowling, 1978). The same is true of sequences of chords (Bharucha \& Krumhansl, 1983; Krumhansl, Bharucha, \& Castellano, 1982). These results suggest that an alteration of a coherent sequence is more easily detected if it disrupts or deviates from the coherent structure than if it preserves it.

In this paper, we assess the detectability of alterations as a function of whether they disrupt the structure of previously coherent text or confer coherence on previously anomalous text. We predict, on the basis of results from other domains in which we have intuitions of coherence, that coherence-disrupting alterations are more easily detected than coherence-conferring alterations. This 
represents an asymmetry in recognition memory, in which discriminative accuracy depends on presentation order. This asymmetry has been observed for melodies (J. C. Bartlett, 1984; Bharucha, 1984a) and sequences of chords (Bharucha \& Krumhansl, 1983; Krumhansl et al., 1982), for which perceived coherence is largely a function of adherence to well-established tonal constraints, and for rhythmic sequences (Bharucha \& Pryor, 1985), for which perceived coherence is largely a function of the implication of an underlying isochronous pulse differentiated on the basis of stress.

We restrict ourselves in this study to coherence and anomaly of pairs of sentences, each sentence being internally coherent and sufficient to establish a meaningful context. In a study phase, subjects were presented with 40 sentence pairs, of which some were coherent vignettes and others were anomalous. An anomalous sentence pair consisted of two sentences describing unrelated situations, causing an interruption after the first sentence. In a subsequent recognition test, subjects judged whether each test pair was identical to the corresponding original pair or was altered by the replacement of one word. The changed word completely altered the meaning of its parent sentence so as to render a previously coherent sentence pair anomalous (coherence-disrupting alteration) or a previously anomalous sentence pair coherent (coherenceconferring alteration). This task required subjects to attempt to detect either the addition or the omission of an unrelated idea within a previously presented sentence pair. We predicted that the addition of an unrelated idea within a sentence pair would be more easily detected than its omission.

A sentence that can be assimilated during testing to an abstract cognitive unit activated during study is more likely to be accepted as original than a sentence that cannot. If the studied sentence pair is coherent, both sentences will activate the same abstract cognitive unit; with a subsequent coherence-distupting alteration, the altered sentence will fail to be assimilated to the originally activated cognitive unit and will easily be detected as new. If the studied sentence pair is anomalous, the two sentences will activate unrelated abstract cognitive units; with a subsequent coherence-conferring alteration, the altered sentence will be assimilated to one of the originally activated cognitive units and will easily be mistaken as old.

Coherent sentence pairs were of the following sort:

(1) The ship pitched wildly in the storm. John hoped the weather would clear up.

For each coherent pair, a corresponding anomalous pair was constructed by replacing one word (the target word) so as to completely change the meaning of one of the sentences. Replacing "weather" with "acne" yields the following anomalous pair:

(1') The ship pitched wildly in the storm. John hoped the acne would clear up.
During the study phase, sentence pair (1) will activate a unifying cognitive unit representing a stormy sea voyage; sentence pair $\left(1^{\prime}\right)$ will activate two abstract cognitive units, one representing a stormy sea voyage and the other an acne problem, with no obvious link between them. If (1) occurs in the study phase and $\left(1^{\prime}\right)$ in the test phase, the second test sentence will not be assimilated to the stormy sea voyage and will easily be detected as new. If $\left(1^{\prime}\right)$ occurs in the study phase and (1) in the test phase, the second test sentence will be assimilated to the stormy sea voyage, making a false alarm likely even though the acne problem was omitted altogether in the test phase.

Another example of a coherent pair is as follows:

(2) The waiter led Dave to a table. The menu was written in French.

Replacing "menu" with "treaty" yields the following anomalous pair:

$\left(2^{\prime}\right)$ The waiter led Dave to a table. The treaty was written in French.

We also looked at alterations in the first sentence of a pair. We predicted that altering the first sentence would make retrieval of the original pair more difficult and hence result in lower accuracy. To the extent that retrieval does occur, we predicted that the asymmetry still should be present, since the coherence-disrupting alteration, whether in the first or second sentence, should find no subsuming cognitive structure that was previously activated. Consider, then, the coherent pair:

(3) The passengers took their seats. The train pulled away from the station.

Replacing "passengers" with "audience" yields the anomalous pair:

(3') The audience took their seats. The train pulled away from the station.

The average reader, given enough time, can fabricate contexts in which an interruption is accommodated. It is not difficult to find contexts in which $\left(1^{\prime}\right),\left(2^{\prime}\right)$, and $\left(3^{\prime}\right)$ are indeed coherent. However, this requires filling in a considerable amount of information in the form of bridging inferences, or images in which both events are present, with the result that more processing time is devoted to anomalous pairs than to coherent pairs (Bellezza \& Bower, 1981; Bower et al., 1979; Haberlandt \& Bingham, 1978; Haviland \& Clark, 1974). To minimize the confounding of coherence with depth of processing in the present experiment, sentence pairs were presented fairly rapidly ( $1 \mathrm{sec}$ per sentence, with $.5 \mathrm{sec}$ between pairs) during study. 


\section{METHOD}

\section{Subjects}

Twenty Dartmouth students participated for course credit.

\section{Apparatus}

Subjects were tested individually on an IBM-XT microcomputer.

\section{Stimuli}

Forty sentence pairs were constructed in each of two versions: coherent and anomalous. In coherent sentence pairs, the two sentences described events or states of affairs that bore an obvious and typical relationship to each other. An anomalous pair was constructed by changing one word (the target) in a coherent sentence pair so that the two sentences no longer bore any obvious relation to each other. The target word occurred in the first sentence in one half of the sentence pairs and in the second sentence in the other half. Sentence length ranged from 5 to 7 words, with one exception of 8 words. The mean length of sentence pairs was 11.26 words (5.68 words for the first sentence, 5.58 words for the second sentence).

The difference between the two versions of each sentence pair was validated by relatedness judgments given by a separate group of 22 subjects. In this validation experiment, two lists were prepared. List A contained, in random order, the coherent versions of half the sentence pairs, picked at random, and the anomalous versions of the other half. List B contained the complementary versions of the sentence pairs in List $A$ in the same order. Half of the subjects were given List $A$ first and then List $B$; the other half were given List B first and then List A. For each pair on each list, subjects indicated how closely related the two sentences were, on a scale of 1 to 7 . The coherent versions (mean rating $=5.80$ ) were judged to be more related than the anomalous versions [mean rating $=1.34, F(1,39)=1705, p<.001]$, with the difference leaning in the same direction for all pairs. Using the Francis and Kucera (1982) frequency count, the means and standard deviations of the frequencies of the target words in English were roughly the same for coherent and anomalous sentence pairs: For the coherent pairs, mean $=17.07$ and standard deviation $=18$; for the anomalous pairs, mean $=17.74$ and standard deviation $=18.96$ (standard error of the difference $=4.01$ )

In the study phase of the main experiment, subjects received 20 coherent and 20 anomalous sentence pairs, presented in a different random order for each subject. In the test phase, half of the originally coherent sentence pairs were the same and half were changed to anomalous, and half of the orginally anomalous sentence pairs were the same and half were changed to coherent.

The two orders of presentation together with the two forms of each sentence pair created four conditions: two "different" conditions (coherence-disrupting and coherence-conferring) and two "same" conditions (coherent and anomalous). A subject saw a sentence pair in only one condition. Subjects were randomly assigned to four groups. The members of each group received the same items in the same conditions, so that among the four groups all items occurred in all conditions. Four buffer items were added at the beginning and four at the end of the study phase but were not included in the test phase.

\section{Procedure}

Each subject was seated in front of the computer in a small room and received the following written instructions:

A series of pairs of sentences will quickly flash onto the computer screen. The first sentence will appear for $1 \mathrm{sec}$ on the middle of the screen: then it will be replaced by the second sentence for $1 \mathrm{sec}$ A brief $(.5 \mathrm{sec})$ line of asterisks will signal the end of the pair. Pairs are not related to each other. You will be tested on comprehension later on. There are 48 sentence pairs. After you have seen them all, the program will stop and the experimenter will give you instructions for the next part of the experiment
The study phase lasted $2 \mathrm{~min}$ and $5 \mathrm{sec}$, after which subjects received the following written instructions for the test phase:

A pair of sentences will appear on the screen. Sometimes it will be identical to what you saw before; sometimes one word will be different, causing a change in meaning. Use the following scale to indicate whether the pair is the same or different:

$1=$ confident it is the same

$2=$ think it is the same but not sure

$3=$ think it is different but not sure

$4=$ confident it is different

Do not deliberate long on an item. If you don't know, guess. Press the number of your choice, then hit return and the next pair will appear. You can backspace to erase an answer before you hit return.

The entire procedure took approximately $10 \mathrm{~min}$

\section{RESULTS}

Table 1 shows the proportion of correct responses, the mean rating score, and the mean area under the receiver operating characteristic (ROC) curve for the coherencedisrupting and coherence-conferring alterations, with the target word in the first and second sentences. Using both subjects and sentence pairs as random factors, proportion correct was significantly higher in the coherencedisrupting condition (.745) than in the coherenceconferring condition $\left[.590 ; \operatorname{minF}^{\prime}(1,42)=4.27\right.$, $p<.05]$. The mean rating score was also significantly higher in the coherence-disrupting condition (3.12) than in the coherence-conferring condition $\left[2.72 ; \operatorname{minF}^{\prime}(1,46)\right.$ $=6.99, \mathrm{p}<.05]$. Thus a significant asymmetry existed on "different" trials. There were no differences between coherent and anomalous sentence pairs on "same" trials (see Table 2).

Table 1

Detection of Single-Word Alterations to Sentence Pairs

Type of Alteration

Target Position Coherence-Disrupting Coherence-Conferring Mean Proportion Correct

\begin{tabular}{llll} 
1st sentence & .650 & .540 & .595 \\
2nd sentence & .840 & .640 & .740 \\
Mean & .745 & .590 & \\
& \multicolumn{1}{c}{ Rating Score* } & & \\
& 2.93 & 2.58 & 2.76 \\
1st sentence & 3.30 & 2.85 & 3.08 \\
2nd sentence & 3.12 & 2.72 & \\
Mean & Area Under ROC & & \\
& .715 & .660 & .688 \\
1st sentence & .807 & .708 & .758 \\
2nd sentence & .761 & .684 & \\
Mean & & & \\
\hline
\end{tabular}

$* I=$ confident same; $4=$ confident different.

Table 2

Performance on "Same" Trials

Type of Sentence Pair

Coherent Anomalous

Proportion Correct $\quad .685 \quad .690$

$\begin{array}{lll}\text { Rating Score* } & 2.04 & 1.99\end{array}$

$* I=$ confident same; $4=$ confident different 
Hit and false alarm rates were computed for each subject at the three decision criteria resulting from the 4-point response scale. Hit rates were computed by collapsing across the two "same" conditions, which were roughly equal in terms of both proportion correct and rating score. Areas under ROC curves were computed for the coherence-disrupting and coherence-conferring alterations, using the proportion of errors on the "different" conditions as false alarms. Performance was significantly more accurate for coherence-disrupting alterations $(.761)$ than for coherence-conferring alterations $[.684 ; \mathrm{F}(1,19)$ $=8.75, \mathrm{p}<.01]$, confirming the asymmetry in terms of accuracy independent of bias.

The proportion of correct responses was significantly higher when the target word was in the second sentence (.740) than when it was in the first $\left[.595 ; \operatorname{minF}^{\prime}(1,56)\right.$ $=4.91, \mathrm{p}<.05$ ]. The mean rating score was also significantly higher when the target word was in the second sentence (3.08) than when it was in the first [2.76; $\left.\operatorname{minF}^{\prime}(1,55)=5.23, p<.05\right]$. Using areas under the ROC curve, performance was significantly more accurate when the target word was in the second sentence $(.758)$ than when it was in the first $[.688 ; F(1,19)=6.50$, $\mathrm{p}<.05]$. When the target word was in the second sentence, the serial position of the target word was highly correlated with the proportion of correct responses collapsed across the two "different" conditions $[r=.463$, $\mathrm{t}(18)=2.21, \mathrm{p}<.05]$. When the target word was in the first sentence, serial position did not affect performance significantly, although the correlation was positive (.263); combining the two groups of items also yielded a nonsignificant positive correlation (.258).

Item length influenced performance, as determined primarily by the length of the second sentence. Correlations with proportion correct collapsed across the four conditions were: $r=-.351$ for sentence pair length $[t(38)$ $=2.31, \mathrm{p}<.05] ; \mathrm{r}=-.406$ for length of the second sentence $[\mathrm{t}(38)=2.74, \mathrm{p}<.01]$; and $\mathrm{r}=-.089$ for length of the first sentence.

\section{DISCUSSION}

Coherence-disrupting alterations were more accurately detected than coherence-conferring alterations. Two sentence-pairs differing in only one word are more easily discriminated from each other if the first is coherent and the second anomalous than vice versa. This implicates abstract cognitive structures in the memory representation of the studied sentence-pairs. Alterations that cannot be assimilated to one of the abstract cognitive structures activated by the studied sentence pair are easily detected; alterations that can be so assimilated are easily confused with the original. Thus, coherencedisrupting foils generated fewer false alarms than did coherence-conferring foils. Hit rates did not differ for coherent and anomalous sentence pairs.

The present results are consistent with those of Graesser et al. (1979), who found higher false-alarm rates for typical information than for atypical information, but only minimal differences in hit rates. They suggest a "script pointer + tag" model, a special instance of the "schema with correction" model of F. C. Bartlett (1932) and Woodworth and Schlosberg (1954): Information that can be reconstructed from more abstract cognitive structures is represented only as a pointer to one of these structures, and only atypical information is tagged. Discriminative accuracy is thus poor for typical information because the specifics are forgotten once the relevant cognitive structure is located. The present experiment does not bear on the question of whether specific information is discarded once an abstract cognitive unit is activated, as Graesser et al. (1979) argue. Nor does it determine whether the abstract cognitive units are incorporated into memory at the time of encoding or are simply reactivated upon retrieval. It does, however, suggest that such structures are embodied in some way in the representation as it is accessed for the subject's decision.

The observed asymmetry mirrors recent results in other domains. Melodies differing in only one tone are more accurately discriminated when the alteration renders a completely tonal melody anomalous than when it renders an anomalous melody completely tonal (J. C. Bartlett, 1984; Bharucha, 1984a). The same is true of chord sequences (Bharucha \& Krumhansl, 1983; Krumhansl et al., 1982). Disruptions of the implied isochrony of previously presented rhythmic sequences are more easily detected than restorations of previously distorted sequences (Bharucha \& Pryor, 1985). In a repeated presentation of a sequence, then, a mistake in the second presentation is more conspicuous than a mistake in the first.

In each domain, our sense of coherence derives from a subsumption of separate events by a more abstract, familiar organizational unit for which a schematic mental representation has been acquired through past exposure. Once activated, abstract cognitive units generate expectations for events that typically follow, facilitating the processing of an entire sequence. More importantly, they permit a hierarchical organization of otherwise disjoint events. Hierarchical organization enables variations on a theme to be recognized as such by permitting either skeletal or elaborated descriptions of the same sequence. Accordingly, hierarchical representations for event sequences have been proposed for each of the domains we have mentioned for which we have intuitions of coherence: text (see Black, 1984), tonal sequences (see Bharucha, 1984b; Deutsch \& Feroe, 1981; Lerdahl \& Jackendoff, 1983), and rhythmic sequences (see Deutsch, 1983; Martin, 1972).

A secondary empirical result in the present experiment was that accuracy was higher when the target was in the second sentence than when it was in the first. Furthermore, when the target was in the second sentence, accuracy increased with serial position. Similar serial position effects have been observed for chord sequences (Bharucha \& Krumhansl, 1983; Krumhansl \& Castellano, 1983). The superior accuracy for targets appearing later rather than earlier suggests that earlier elements serve as better retrieval cues than do later ones. With later altera- 
tions, more information is available for retrieving the memory representation of the learned sentence pair, and subjects are less likely to guess. To the extent that the representation of the original sentence pair has been retrieved, however, the asymmetry is manifest.

In addition to the influence of abstract cognitive units on memory judgments, the observed asymmetries suggest that in a same-different task for sequences, the judgment process does not consist of a symmetric matching of the test sequence with the representation of the studied sequence. If this were so, the omission of an unrelated idea in the test sequence would be just as easily detected as the introduction of a new one. A coherence-disrupting alteration introduces an unrelated idea, whereas a coherence-conferring alteration omits one, and the former is more easily detected than the latter. The judgment process thus plausibly consists of checking the test sequence for inclusion in the representation of the studied sequence, but not vice versa. Even if, as in Graesser et al. (1979), atypical information is given a special tag in memory, its omission in a recognition test is less noticeable than the presentation of new atypical information, unless of course the tagged information constitutes the "point" of the story (see Bower et al., 1979).

J. C. Bartlett (1984) argues for an account of memory asymmetries (for melodies) that steers clear of positing structures in long-term memory. According to his account, which is similar to Garner's (1970) characterization of good forms, a coherent sequence delimits a smaller space of alternatives than does an anomalous sequence. Fewer alterations are accepted as structure-preserving variations-" "structure" referring here to stimulus structure, not cognitive structure. This view reflects an ecological approach, according to which familiarity of structure influences performance not because of schematic structures in long-term memory but because the organism is attuned to picking up invariant information. Alterations that render an anomalous sequence coherent are included within the delimited space, but alterations that render a coherent sequence anomalous are not. This account is functionally equivalent to the present account, with the difference that it does not posit a mechanism by which the space of alternatives is delimited. The activation of abstract cognitive structures is a plausible mechanism that represents the space of acceptable alternatives as psychological space. J. C. Bartlett's (1984) analysis, as well as ours, implies that a recognition judgment in this experiment is based on a decision as to whether the sentences of the test pair are a subset of the semantic space delimited by the studied pair. Thus the addition of an anomaly is more easily detected than its omission.

\section{REFERENCES}

BarTletr, F. C. (1932). Remembering. New York: Cambridge University Press.

BartLeTt, J. C. (1984). Cognition of complex events: Visual scenes and music. In W. R. Crozier \& A. J. Chapman (Eds.), Cognitive processes in the perception of art. Amsterdam: North-Holland.

BELLEZZA, F. S., \& BowER, G. H. (1981). The representational and processing characteristics of scripts. Bulletin of the Psychonomic Society, 18, 1-4.

Berman, J. S., Read, S. J., \& Kenny, D. A. (1983). Processing inconsistent social information. Journal of Personality \& Social Psychology, 45, 1211-1224.

BHARUCHA, J. J. (1984a). Anchoring effects in music: The resolution of dissonance Cognitive Psychology, 16, 485-518.

BHARUCHA, J. J. (1984b). Event hierarchies, tonal hierarchies, and assimilation: A reply to Deutsch and Dowling. Journal of Experimental Psychology: General, 113, 421-425.

Bharucha, J, J., \& KRUMhansl, C. L. (1983). The representation of harmonic structure in music: Hierarchies of stability as a function of context. Cognition, 13, 63-102.

BHARUCHA, J. J., \& PrYoR, J. (1985). Asymmetric discrimination of rhythmic sequences and anomalous variants. Manuscript submitted for publication.

BLACK, J. B. (1984). Understanding and remembering stories. In J. R. Anderson \& S. M. Kosslyn (Eds.), Tutorials in learning and memory: Essays in honor of Gordon Bower. New York: Freeman.

Bower, G. H., Black, J. B., \& TURNER, T. J. (1979). Scripts in memory for text. Cognitive Psychology, 11, 177-220.

Castellano, M. A., Bharucha, J. J., \& Krumhansl, C. L. (1984). Tonal hierarchies in the music of North India. Journal of Experimental Psychology: General, 113, 394-412.

COHEN, M. E., \& CARR, W. J. (1975). Facial recognition and the von Restorff effect. Bulletin of the Psychonomic Society, 6, 383-384.

Cuddy, L. L., Cohen, A. J., \& Mller, J. (1979). Melody recognition: The experimental application of musical rules. Canadian Journal of Psychology, 33, 148-157.

DEuTSCH, D. (1983). The generation of two isochronous sequences in parallel. Perception \& Psychophysics, 34, 331-337.

Deursch, D., \& FEROE, J. (1981). The internal representation of pitch sequences in tonal music. Psychological Review, 88, 503-522.

DowLING, W. J. (1978). Scale and contour: Two components of a theory of memory for melodies. Psychological Review, 85, 341-354.

FranCIS, W. N., \& KUČERA, H. (1982). Frequency analysis of English usage: Lexicon and grammar. Boston: Houghton Mifflin.

GarNer, W. R. (1970). Good patterns have few alternatives. American Scientist, 58, 34-42.

Graesser, A. C., Gordon, S. E., \& Sawyer, J. D. (1979). Memoty for typical and atypical actions in scripted activities: Test of a script pointer + tag hypothesis. Journal of Verbal Learning \& Verbal Behavior, 18, 319-332.

HaherLANDT, K., \& Bingham, G. (1978). Verbs contribute to the coherence of brief narrative passages: Reading related and unrelated sentence triplets. Journal of Verbal Learning \& Verbal Behavior, 17, 419-425.

Hastie, R., \& Kumar, P. A. (1979). Person memory: Personality traits as organizing principles in memory for behaviors. Journal of Personality \& Social Psychology, 37, 25-38.

Haviland, S. E., \& ClaRK, H. H. (1974). What's new? Acquiring new information as a process in comprehension. Journal of Verbal Learning \& Verbal Behavior, 13, 512-521.

Johnson, J. T., \& JudD, C. M. (1983). Overlooking the incongruent: Categorization biases in the identification of political statements. Journal of Personality \& Social Psychology, 45, 978-996.

Krumhansl, C. L., Bharucha, J. J., \& Castellano, M. A. (1982). Key distance effects on perceived harmonic structure in music. Perception \& Psychophysics, 32, 96-108.

Krumhansl, C. L., \& Castellano, M. A. (1983). Dynamic processes in music perception. Memory \& Cognition, 11, 325-334.

Krumhansl, C. L., \& Kessler, E. J. (1982). Tracing the dynamic changes in perceived tonal organization in a spatial representation of musical keys. Psychological Review, 89, 334-368.

LehNert, W. G. (1981). Plot units and narrative summarization. Cognitive Science, 5, 293-331.

LERDAHL, F., \& JACKENDOFF, R. (1983). A generative theory of tonal music. Cambridge: MIT Press. 
Light, L. L., Kayra-Stuart, F., \& Hollander, S. (1979). Recognition memory for typical and unusual faces. Journal of Experimental Psychology: Human Learning \& Memory, 5, 212-228.

Martin, J. G. (1972). Rhythmic (hierarchical) versus serial structure in speech and other behavior. Psychological Review, 79, 487-509.

RUMELHART, D. E. (1977). Understanding and summarizing brief stories. In D. G. Bobrow \& A. M. Collins (Eds.), Basic processes in reading: Perception and comprehension. Hillsdale, NJ: Erlbaum.

SCHANK, R., \& ABELSON, R. P. (1977). Scripts, plans, goals, and understandings: An inquiry into human knowledge structures. Hillsdale, NJ: Erlbaum.
Thorndyke, P. W. (1977). Cognitive structures in comprehension and memory of narrative discourse. Cognitive Psychology, 9, 77-110.

Woll, S. B., \& GRAESSER, A. C. (1982). Memory discrimination for information typical or atypical of person schemata. Social Cognition, 4, 287-310.

WOODWORTh, R. S., \& SCHLOSBeRG, H. (1954). Experimental psychology. New York: Holt, Rinehart \& Winston.

(Manuscript received December 19, 1984; revision accepted for publication July 16, 1985.) 